Revue d'histoire du XIXe siècle

Société d'histoire de la révolution de 1848 et des

révolutions du XIXe siècle

\title{
Vincent DUCLERT, Rémi FAVRE et Patrick FRIDENSON [dir.], Avenirs et avant-gardes en France (XIXe-XXe siècles). Hommage à Madeleine Rebérioux
}

Paris, Éditions La Découverte, 1999, 439 p.

Frédéric Chauvaud

\section{OpenEdition}

\section{Journals}

Édition électronique

URL : http://journals.openedition.org/rh19/333

DOI : $10.4000 /$ rh19.333

ISSN : $1777-5329$

Éditeur

La Société de 1848

Édition imprimée

Date de publication : 1 décembre 2001

Pagination : $272-273$

ISSN : 1265-1354

Référence électronique

Frédéric Chauvaud, "Vincent DUCLERT, Rémi FAVRE et Patrick FRIDENSON [dir.], Avenirs et avantgardes en France (XIXe-XXe siècles). Hommage à Madeleine Rebérioux », Revue d'histoire du XIXe siècle [En ligne], 23 | 2001, mis en ligne le 15 octobre 2002, consulté le 22 septembre 2020. URL : http:// journals.openedition.org/rh19/333; DOI : https://doi.org/10.4000/rh19.333

Ce document a été généré automatiquement le 22 septembre 2020.

Tous droits réservés 


\section{Vincent DUCLERT, Rémi FAVRE et Patrick FRIDENSON [dir.], Avenirs et avant-gardes en France (XIXe- XXe siècles). Hommage à Madeleine Rebérioux}

Paris, Éditions La Découverte, 1999, 439 p.

Frédéric Chauvaud

Livre d'hommage et plaidoyer pour une certaine histoire sociale qui restitue aux acteurs un rôle essentiel sans pour autant être exclusif, cet ouvrage réunit une trentaine de contributions réparties en six approches thématiques. Toutes ne concernent pas le grand XIX ${ }^{e}$ siècle, mais toutes méritent d'être lues. Une première entrée, passionnante, s'arrête sur l'itinéraire de "la citoyenne" pour reprendre le titre du portrait proposé par Pierre Vidal-Naquet, qui relate en quelques pages alertes une amitié née en 1958, au moment où la guerre d'Algérie suscitait la "vigilance universitaire". Dans un autre registre Marianne Schaub scrute avec méticulosité et sympathie l'atelier de l'historienne, tandis que Béatrice Slama relate de quelle façon, dans le cadre de l'interdisciplinarité revendiquée, le travail sur les femmes s'est élaborée à Vincennes dans les années 1970, avec la mise sur pied d'un séminaire "ouvert" dont "un des moments forts" a été l'étude du discours d'Hubertine Auclert au troisième congrès ouvrier de 1879. La deuxième entrée s'attache aux femmes et aux mouvements de femmes. Michèle Riot-Sarcey, revenant sur ses propres travaux, s'intéresse en quelque sorte aux conséquences de l'histoire des femmes et des gender studies qui ont joué un rôle majeur pour l'analyse de la construction des catégories sociales, sources d'identité. Nicole Savy, dans un petit texte dense et ramassé, traite de la question de l'universalité et conclue sur l'impérieuse nécessité de défendre les droits des femmes sur tous les terrains menaçants. Yolande Cohen donne un texte ambitieux qui inscrit les gender studies dans le sillage des théories de l'agency, puis évoque le passage de l'histoire des femmes à l'histoire des genres, pour offrir, à partir du cas 
québécois, une réflexion sur les identités sociales et les identités nationales. Le second $\mathrm{XX}^{\mathrm{e}}$ siècle est au cœur de la démonstration de Christophe Charle analysant les dynamiques et les freins de la présence des femmes dans l'enseignement supérieur. Marie-Noëlle Thibault offre les premiers éléments d'une réflexion sur la place des femmes dans le rapport Villermé.

Le troisième temps de l'hommage à Madeleine Rebérioux évoque le travail de la culture et s'ouvre par une passionnante synthèse de Marie-Claude Genet-Delacroix sur les liaisons entre académisme et avant-garde dans la peinture française au XIX ${ }^{e}$ siècle. Il s'agit d'un examen des pratiques et de l'expérience artistique. Christophe Prochasson s'interroge sur les façons de fonder la renommée d'une ville, en l'occurrence le Paris 1900, qui s'est évertué à forger un mythe, celui du brio. C'est un autre biais qu'a retenu Gilles Candar qui restitue au cours des années rétives que sont les années 1920, la critique littéraire dans la Nouvelle revue socialiste. Philippe Gumplowicz livre une contribution pétillante sur la cause du jazz et la ferveur des premiers amateurs. Plus loin du XIX ${ }^{e}$ siècle, Lucette Valensi analyse finement la littérature et la critique sociale dans l'Algérie post-coloniale.

L'enjeu du travail constitue le quatrième temps de ce livre collectif. Maïté Bouyssy donne une contribution ciselée et érudite sur le "tout politique" de la montre industrielle, tandis que Gérard Noiriel revient sur certains aspects de la lutte des ouvriers de Longwy contre la restructuration de la sidérurgie française, et que Patrick Fridenson affirme que l'avenir constitue le fonds de commerce des patrons et s'empare du cas de Pierre Lefaucheux.

Cinquième entrée : nations et guerre entre socialisme et internationalisme. MarieLouise Goergen suit les correspondants de presse à la fin du XIX ${ }^{e}$ siècle. S'ils peuvent servir de liaison entre socialisme français et socialisme allemand, leur recrutement pose un véritable problème pour les responsables des journaux et des revues. Rémi Fabre s'attache à l'itinéraire de Gustave Rouanet, fils de menuisier de la Montagne noire et député de Paris. À l'occasion de l'affaire Toqué-Gaud qui en 1903 au Congo avait exécuté à la dynamite un malheureux, il engage dans l'Humanité une véritable campagne contre la "barbarie coloniale". Charles Heimberg procède à une relecture de l'Armée nouvelle de Jaurès en s'intéressant à sa réception par le mouvement ouvrier suisse-romand qui pose le problème de la conscription de milice. Tandis que JeanJacques Becker se met en quête de l'opinion publique française en 1917 face à la révolution d'Octobre et conclue qu'elle est finalement très proche de ce qu'elle fut en 1914 où le "sentiment national" l'emporta, François Delpa étudie les positions de Léon Blum face à l'Allemagne nazie.

La dernière division de l'hommage à Madeleine Rebérioux embrasse les questions relatives à l'engagement politique et aux choix éthiques. Vincent Duclert écrit avec élégance, point de départ pour cerner l'engagement démocratique, que "l'affaire Dreyfus est incontestablement une événement qui ne se referme pas sur le passé et qui ouvre au contraire sur l'avenir des sociétés modernes". Ensuite Gérard Baal traite des "jeunes radicaux" --la paternité de l'expression reviendrait à Marc Sangnier-- au cours de la période 1910-1914, dans un contexte de crise de la "République radicale". Quant à Lucien Mercier, s'il s'intéresse également à la jeunesse, il a porté, dans une étude très documentée, son regard sur le scoutisme rouge qui propose la vision neuve d'une enfance militante. Les instituteurs, que Jacques Girault connait si bien, sont l'objet d'une recherche consacrée aux itinéraires de militants responsables du Syndicat national des instituteurs pendant la Seconde Guerre mondiale. René Gallisot referme le 
volume en en étudiant la revue Politique aujourd'hui, née de Mai 1968 et qui, à partir de janvier 1969, tient la gageure d'être mensuelle.

Au total, un livre à la fois érudit et chaleureux qui propose une esquisse panoramique des recherches en cours en histoire contemporaine. 